\title{
Notes du terrain : Incidence du super typhon Yolanda sur le programme d'immunisation systématique aux Philippines
}

\author{
Shane $A^{1 *}$ \\ ${ }^{1}$ Agence de la santé publique du Canada, Centre de l'immunisation et des maladies respiratoires infectieuses, \\ Ottawa (Ontario) \\ *Auteure-ressource : Amanda.Shane@phac-aspc.gc.ca
}

Le 8 novembre 2013, le typhon le plus violent jamais enregistré a frappé Guiuan, aux Philippines. Avant de se dissiper le 11 novembre, le typhon, qui progressait vers l'ouest, a traversé 41 provinces. On estime que 16 millions de personnes ont été touchées ( $17 \%$ de la population totale), dont 4,1 millions ont été déplacées, 6300 sont décédées et 1061 ont été portées disparues (1,2). Les dégâts considérables causés aux infrastructures, aux systèmes d'approvisionnement en eau et en électricité, ainsi qu'aux installations de communication s'expliquent par des vents violents (ayant dépassé les $300 \mathrm{~km} / \mathrm{h}$ dans certaines régions) combinés à des ondes de tempête inattendues le long de certaines zones côtières, similaires aux effets d'un tsunami (1).

Par l'entremise du Réseau mondial d'alerte et d'action en cas d'épidémie (3), I'Organisation mondiale de la Santé (OMS) a demandé l'aide à l'Agence de la santé publique du Canada dans le domaine de la santé publique. J'ai été l'un des épidémiologistes qui ont fourni cette aide. Avant mon arrivée, quatre de mes collègues de l'Agence avaient déjà effectué des missions de durée variable sur le terrain entre novembre 2013 et février 2014. Ils ont fourni différentes formes d'orientation et de soutien à une unité d'aide d'urgence dans les domaines de la surveillance et de l'intervention en cas d'éclosion de maladie. Lorsque je suis arrivé fin février 2014 (presque quatre mois après la catastrophe), l'antenne de l'OMS aux Philippines, après la phase d'intervention, avait commencé à passer à la phase de rétablissement et, donc, à se concentrer sur les initiatives et les programmes à long terme.

À mon arrivée, j'ai été désigné agent de coordination de l'OMS pour le Programme élargi de vaccination dans la région 8 , la plus durement touchée. Mon objectif principal était d'évaluer les effets du typhon sur le programme d'immunisation systématique dans la région. II s'agissait là de poursuivre le travail entamé par un expert-conseil de I'U.S. Centers for Disease Control and Prevention qui avait effectué une mission d'une semaine dans la région à la fin du mois de février 2014. Entre février et avril 2014, je me suis rendu dans 54 établissements situés dans 35 municipalités. La majorité des établissements a signalé que le typhon avait provoqué des dommages structurels et la rupture de l'alimentation électrique. Par conséquent, une grande partie des établissements a dû interrompre ses activités d'immunisation systématique. La durée médiane de la rupture de l'alimentation électrique était de huit semaines, mais un sous-ensemble de zones n'a pas pu procéder à l'immunisation systématique pendant plus de trois mois. Cependant, au moment des visites sur place, tous les établissements avaient réussi à reprendre leurs activités d'immunisation systématique.

Cette évaluation a permis de déterminer les critères clés pour procéder au rétablissement d'un programme d'immunisation. Parmi ces critères, il y avait notamment l'importance du matériel de préservation de la chaîne du froid résistant aux catastrophes naturelles, des plans d'urgence bien définis pour le maintien de la chaîne du froid et des processus clairs pour fournir du matériel d'immunisation ( $p$. ex. un registre de remplacement) et une réserve de vaccins de remplacement en cas de catastrophe. L'OMS et le ministère de la Santé des Philippines se serviront des résultats de cette évaluation pour relever les défis cernés et renforcer le programme d'immunisation systématique.

J'ai éprouvé quelques difficultés au cours de cette mission, notamment pour les aspects suivants : comprendre le système de santé publique complexe des Philippines; nouer des relations constructives avec les intervenants des 
principales organisations nationales/régionales/provinciales gouvernementales et de certaines organisations non gouvernementales dans un laps de temps très court; et composer avec les limites découlant des restrictions logistiques. Toutefois, malgré cela, mon impression générale a été très positive. J'ai été impressionné par la résilience et le professionnalisme des travailleurs de la santé philippins que j'ai rencontrés lors de mes déplacements dans la région. Bon nombre de ces personnes avaient été directement touchées par le typhon et avaient perdu des membres de leur famille, des amis ou des collègues dans la catastrophe. Malgré cela, j'ai pu observer qu'elles étaient fermement résolues à ce que les enfants de leur collectivité aient accès au Programme élargi de vaccination.

\section{Références}

(1) Humanitarian Response Philippines. Multi-cluster/sector initial rapid assessment: Philippines typhoon Yolanda Haiyan.

https://philippines.humanitarianresponse.info/system/files/documents/files/20131129_MIRA_ Report_-_Philippines_Haiyan_FINAL_1.pdf

(2) National Disaster Risk Reduction and Management Council. NDRRMC update: Updates re: The effects of typhoon "Yolanda" (Haiyan).

Http://www.ndrrmc.gov.ph/attachments/article/1177/Update\%20Effects\%20TY\%20YOLANDA \%2017\%20April\%202014.pdf

(3) Organisation mondiale de la Santé. Réseau mondial d'alerte et d'action en cas d'épidémie (GOARN). http://www.who.int/csr/outbreaknetwork/fr/ 